\title{
Rethinking dentistry and dental teaching
}

\author{
Jose Leopoldo Ferreira Antunes ${ }^{1}$ (1) \\ ${ }^{1}$ School of Public Health, University of São Paulo, São Paulo, Brazil \\ ${ }^{2}$ Federal University of Rio Grande do Sul, Rio Grande do Sul, Brazil \\ ${ }^{3}$ Department of Public Health and Infectious Diseases, Sapienza University of Rome, Rome, Italy
}

Amanda Ramos da Cunha²

Stefano Petti ${ }^{3}$

Correspondence

Jose Leopoldo Ferreira Antunes, School of Public Health, University of São Paulo, 715 Dr. Arnaldo Av, São Paulo, 01246-904, Brazil.

Email: leopoldo@usp.br

\section{1 | SPOTLIGHT ON ORAL HEALTH - THE 2019 LANCET SERIES}

The launch of the Lancet Series on oral health offers a unique opportunity to rethink dentistry and dental teaching.

The first paper (Peres et al., 2019) of the Series delimitated the subject. Oral diseases are major and long-standing public health problems worldwide. They affect large population contingents: toddlers and children, adolescents, adults, and older people: all of them have their share, no age group is spared. Dental caries, periodontal disease, and other oral disorders are largely preventable; notwithstanding, they remain among the most prevalent health conditions. Their treatment consumes enormous financial costs to the individuals, their families, and community health systems. The burden of disease is enormous in terms of pain, infection, quality of life, and school and work absenteeism. Clinical, laboratory, and epidemiological knowledge on oral diseases has accumulated over time, but has not allowed controlling them, nor has reduced severe inequalities in their distribution in contemporary societies. A higher burden of disease falls on the more impoverished socioeconomic strata within countries. Between societies, the problem is higher in low- and middle-income countries.

The second paper (Watt et al., 2019) proposed a way forward. Oral health has been globally neglected for too long; radical and decisive action is urgently needed. Facing such an immense challenge demands a comprehensive plan. The authors formulated the strategy with eight recommendations, encompassing from the improvement of surveillance in oral health to investing in population-wide policies aimed at the underlying causes of diseases. These tasks go side by side with promoting an appropriate exposure to fluoride, advancing the agenda of dental research, and fostering the advocacy of oral health at the population level. Keep the page with the panel of recommendations; it will be useful to check on afterward for future accomplishments.
Individually treating those already affected by dental diseases will never suffice to control dental diseases, without addressing the commercial interests that act against oral health. The authors commanded explicitly for upstream policies directed toward the primary determinants of oral health. The recommendations contemplated taxing the sugar industry and regulating the advertising of its products, along with other deleterious exposures, as tobacco and alcohol consumption. The global market of commercial baby foods and drinks is of particular interest. In addition to increasing caries risk, processed foods with high sugar content promote an enduring preference for sweetness and may contribute to obesity and overweight.

The strategy is compelling; its proposition goes in line with the new definition of oral health (Glick et al., 2017), approved in 2016 by the General Assembly of the FDI World Dental Federation, which brought forth the multifaceted nature and full scope of oral health. Effectively ending the neglect of oral health worldwide will demand a concerted societal effort. The dental public health approach must integrate public and private efforts; it must include preventive population-based initiatives and patient-centered care. For dentistry and dental teaching, the quest for a new oral health demands rethinking their premises and aims.

Dentistry and dental teaching have followed an interventionist approach, which is dominated by a technical philosophy that dates back to the surgical origins of the profession. Reforming concepts, methods, and practices of dentistry and dental training is imperative if the aim is to change oral health. Accomplishing the new definition of oral health is not compatible with the training of specialists exclusively aimed at treating diseases already installed. The profession will have to deal with the determinants of oral health at the population level.

The new dentists should overcome the perspective of working in isolation and qualify themselves to participate in teams, integrating the efforts of different classes of health professionals. 
Interprofessional collaboration is crucial to improve the surveillance of dental indicators and indices; it is also necessary to strengthen primary care in oral health. The new dentists must have a say in the training of the dental workforce, thus integrating dental nurses, hygienists, and others. The interdisciplinary dialogue has to include other health professionals, from recording clerks, who assist the examiner in oral health surveys, to pediatric practitioners, who usually examine the children's oral cavity even earlier than any dentist. Faced with so many significant changes that are indicated by the panel of recommendations, strategic efforts may need to be more concentrated in critical initiatives. Restructuring dental training needs to receive attention consistent with its relevance and potential impact on all the areas of the proposed reform.

Moving to the new paradigm of oral health is not an easy endeavor; the panel of recommendations of the Lancet series had eight items, which will have to be implemented and monitored. They will also have to be coordinated. Well-orchestrated pro-change initiatives can be undermined if they are not aligned with other strategic areas. If set in a context of easy access to sugary products, a universal and equitable oral health care system, may have reduced its impact on oral health. The debate on how to implement the oral health agenda has already begun. Kearns and Bero (2019) addressed the theme of financial conflicts of interest between dentistry and the sugar industry and made recommendations for the future of the profession. Beaglehole and Beaglehole (2019) assessed different strategies to foster global oral health and discussed the perspectives of integrating it into health programs aimed at non-communicable diseases or keeping it independent.

\section{2 | CHALLENGES IN RETHINKING DENTISTRY}

The road that takes to a comprehensive, equitable, and effective dental healthcare for all is even harder than how it may seem from the oral health 2019 Lancet series. Many of the issues to consider in rethinking dentistry and dental teaching are mountains to climb. We focused on some challenges to meet the goal of health for all.

\subsection{Countering the power and influence of Big Sugar}

Undeclared and opaque conflicts of interest between the sugar industry and some dental organisations and academic institutions need to be addressed.

(Watt et al., 2019)

The problem of conflicts of interest affects many scientific societies and academics (Kearns \& Bero, 2019). However, there is something more. The majority of dental healthcare providers, public dental health officers, and policymakers without conflicts of interest are unaware that the lobbying activity of Big Sugar ended up in drastically changing what is known and what is no longer known about dental caries dietary risk factors.

According to the widely accepted "acid theory" (van Houte, 1994), that evolved into "nonspecific plaque hypothesis" and then to "ecological plaque hypothesis" (Pitts et al., 2017), caries is due to frequent low $\mathrm{pH}$ pulses due to carbohydrate (mono-, disaccharides, and starch) fermentation by oral bacteria at the tooth-dental plaque interface responsible for the selection of acidogenic-aciduric bacteria and enamel demineralization (Marsh, 2018). In 1994, van Houte published an influencing review in the Journal of Dental Research, reporting the results of more than 20 years of research on caries etiology (van Houte, 1994). According to this review, although the "acid theory" was correct, a specific group of microorganisms and a specific sugar have a pivotal role in the initial stages of enamel caries, namely, mutans streptococci and sucrose. Streptococci and other oral bacteria produce extracellular polymers from fructose and glucose-the two monosaccharides contained in sucrose (which is a disaccharide), known as fructans and glucans. These polymers are linear and do not interfere with the typical dental plaque structure, characterized by tight bacterial cell masses in the deeper layers. In normal conditions, the availability of nutrients to bacteria in these layers, even during meals, is minimal, and $\mathrm{pH}$ at the tooth-plaque interface is consequently close to neutral values (Marsh, 2018). However, mutans streptococci produce a particular glucan from sucrose that is insoluble and highly branched. A high content of this particular glucan in dental plaque increases the strength of its adhesion on hard surfaces and, very importantly, its $\mathrm{pH}$ lowering ability. Such an increased $\mathrm{pH}$ lowering ability of dental plaque is due to the particular characteristic of this insoluble glucan, that increases the interbacterial distance, thus increasing plaque porosity, and allowing large amounts of dietary fermentable carbohydrates, sugars, and starch without exception, to reach the deeper bacterial layers close to the enamel, and increasing sugar availability to acidogenic-aciduric bacteria, mutans and non-mutans streptococci, lactobacilli, and bifidobacteria without exception. This mutans streptococcisucrose-specific stage often precedes the nonspecific "ecological plaque hypothesis" stage, when different bacterial species use different carbohydrates as substrates, thus causing the development of the caries lesion. This simple, clear, and intriguing model can explain why all fermentable carbohydrates and fermenting bacteria can be considered cariogenic and why, at the same time, sucrose and mutans streptococci are more relevant than the remainder in initiating the enamel caries process (Zero, 2004). This model does not exclude the chance that caries can develop without mutans streptococci in plaque and sucrose in diet, but suggests that this event is less likely (van Houte, 1994).

In 2000, van Houte published another review in an influential dental journal, concluding that it was premature to consider food starches in modern diets as safe for teeth (Lingström, Houte, \& 
Kashket, 2000). Two particular characteristics made this review different from the former. Namely, van Houte was not the only author, and the research was supported by the sugar company Mars Incorporated, USA. Why would a sugar company fund a review on the detrimental effect of starch on dental health?

This study was aligned with the Big Sugar strategy of promoting the nonspecific caries etiology theory. Namely, all fermentable carbohydrates and all fermenting oral bacteria yield some cariogenic potential and foods like bread, potatoes, even grapes, and apples can be cariogenic in some circumstances. Thus, since all carbohydrates are cariogenic and are an essential component of the human diet and there are no safe alternatives, caries prevention programs should not be based on dietary counseling (van Loveren, 2009).

In 2015, the World Health Organization (WHO) published the Guideline on Sugars Intake for Adults and Children and recommended limiting free sugars to no more than $5 \%$ total energy intake to protect oral health throughout the life course. Free sugars are the added monoand disaccharides and the sugars in honey, syrups, fruit juices, and fruit juice concentrates (Moynihan, Makino, Petersen, \& Ogawa, 2018). Authors funded by the ILSI immediately criticized this recommendation as being not evidence-based (Erickson \& Slavin, 2015) and continued their battle against nutrition guidelines (Erickson, Sadeghirad, Lytvyn, Slavin, \& Johnston, 2017). Nevertheless, this criticism did not produce any guideline revision, apparently suggesting that Big Sugar lost this battle. However, it was not so.

Even today, some public dental health experts are not aware that sucrose, as Ernest Newbrun stated as early as in 1969, is the arch-criminal of dental caries (Newbrun, 1969). Indeed, a survey with 45 dental caries experts from 20 European countries reported that as many as $40 \%$ of them disagreed with the Newbrun's sentence, $18 \%$ were neutral, and only $42 \%$ (including one of the authors of the present article) were in agreement (van Loveren \& Duggal, 2004).

The paradox is this. In the 1960s, 1970s, and 1980s, almost all public dental health experts were aware of the specific role of sucrose in caries initiation, while from the late 1980s and even today, sucrose is considered just one of the many sugars that need to be controlled to prevent caries (Pitts et al., 2017). The WHO guidelines are fated to fail in the long term if most public dental health experts from all over the world cannot discriminate between the high cariogenic activity of sucrose compared to the other mono- and disaccharides, because people who base their daily free sugar intake on sucrose are at high risk of caries, even if this intake is below the recommended limit of $5 \%$ of total energy.

Incidentally, the ILSI is currently controlling dental research, but in an unclear way. Indeed, the aforementioned ILSI publication of 2009 (van Loveren, 2009) was sponsored by the ILSI Europe Oral Health Task Force, which is funded by four industry members: the DSM, a multinational active in the field of nutrition, Mars, Südzucker, and GlaxoSmithKline (GSK). Although this task force is now over, GSK remains an ILSI funder through one of its many branches, the ILSI Health and Environment Sciences Institute (ILSI-HESI). To make things even more clouded, the ILSI-HESI changed its name into HESI, but did not change its state of an ILSI branch. In addition,
GSK, along with Pepsico, Coca-Cola, Danone, Nestle, and DSM are funding the laboratory of Dr. Slavin, author of the aforementioned criticism against the WHO guideline recommendations on sugar intake (Erickson et al., 2017; Erickson \& Slavin, 2015), a project that was explicitly funded by the Technical Committee on Dietary Carbohydrates of the North American branch of ILSI.

GSK is currently a corporate member and/or sponsor of many scientific societies, including the European Organisation for Caries Research, European Association of Dental Public Health, Association for Dental Education in Europe, European Federation of Periodontology, Platform for Better Oral Health in Europe, and many other similar organizations. Although GSK is also a brand of oral hygiene products, it also participates in the sugar industry, through its branch GSK Consumer Healthcare merged with Hindustan Unilever Ltd, a branch of another important company involved in the sugar industry. Thus, both Big Sugar and Big Pharma are jointly supporting the program of ILSI and its branches to make dental healthcare providers unaware of the central role of sucrose in caries development. The reason is clear: $5 \%-10 \%$ of total calorie intake from carbohydrates according to the various nutrition guidelines (Erickson et al., 2017) is an important business if no difference is placed between sucrose and starch, fruit, milk, and other carbohydrates. It is probably for this reason that the position of Unilever regarding sugar reduction (available at https://www.unilever.com/Images/unilever-position-on-sugar-reduc tion_tcm244-423167_en.pdf) is softer than the ILSI position.

\section{2 | Inadequate coalescence and leadership among global oral health actors. The case of Traumatic Dental Injuries}

The current state of global oral health is... not only a result of external factors... but also related to inadequate coalescence and leadership among global oral health actors.

(Watt et al., 2019)

The disentanglement between global oral health actors produced one of the most paradoxical situations in the history of public health, and it relates to Traumatic Dental Injuries (TDI).

The Global Burden of Disease Study (GBD) is a comprehensive research program that produces relevant epidemiological data on the most important diseases and injuries. According to the GBD, dental caries in permanent teeth was the most prevalent disease in the world in 2015, affecting more than two billion people. Caries in deciduous teeth and periodontitis ranked twelfth and fourteenth, respectively; together, they affected more than half a billion individuals (GBD 2015 Disease and Injury Incidence and Prevalence Collaborators, 2016). A group of oral health experts from all over the world provided the data on oral health (Kassebaum et al., 2017).

Now, what would one say if there was another oral condition, not included in the GBD, which affects as many as one billion people 
in the world and is homogeneously spread in the six WHO Regions? This oral condition exists, and it is TDI, whose global prevalence was estimated to be $15 \%$ (Petti, Glendor, \& Andersson, 2018). Traumatic dental injuries would rank fifth in the GBD list of most prevalent diseases and injuries, but this condition is so neglected that even the GBD did not consider it. Similarly, remarkable public health organizations worldwide, including the $\mathrm{WHO}$ and Centers for Disease Control and Prevention, do not include TDI in their lists of oral health topics. And it is not over yet, as even the International Classification of Diseases (ICD), the acknowledged diagnostic classification standard for clinical and research purposes, does not consider TDI as a specific disorder, and excluded some types of TDI, such as infraction, concussion, and subluxation (Petti, Andreasen, et al., 2018).

TDI unawareness causes diagnostic confusion, misclassification, and even non-classification in healthcare settings such as the emergency departments (EDs), where most healthcare workers are not skilled in dentistry. People affected by TDI generally refer to dental clinics and hospitals, which, however, are not uniformly distributed in the territory, and are often unavailable during holidays, weekends and night time. From the public health standpoint, misclassification in EDs induces an underestimation of the TDI burden that results in TDI unawareness among public health officers and policymakers. In consequence, they do not allocate adequate resources for TDI prevention and treatment, and they are not encouraged to design adequate TDI control policies. Treating this condition is time-consuming and expensive; a study in Denmark estimated the demand of 2,000,000-5,000,000 USD per million inhabitants annually (Borum $\&$ Andreasen, 2001). From an individual standpoint, this unawareness among ED healthcare workers results in inequalities in oral health status between the rich and the poor, because EDs are essential points of entry into the public healthcare system for disadvantaged people. Indeed, while the rich are treated in the private sector, the poor need TDI diagnosis in EDs to get free access to proper dental care in the public sector (Alnaggar \& Andersson, 2015).

Like many oral diseases, TDI shares common risk factors with several non-communicable diseases and injuries. Indeed, excluding large overjet, a specific dental-related risk factor (Petti, 2015), the leading causes of this condition are related with behavior-falls, physical leisure activity, sport playing, collisions, traffic accidents, and violence (Andersson et al., 2019)-whereas obesity is another important risk factor (Corrêa-Faria \& Petti, 2015).

Units dedicated to TDI control must, therefore, be designed and included in broader programs aimed at the prevention and the control of violence on children, road, home, and sports accidents, and injuries occurring during outdoor play.

\section{3 | Focusing on the social determinants of health-the Inverse Equity Hypothesis}

Recognition is growing for the need to move... to policy initiatives that tackle oral health inequalities at the structural level, focusing on the social determinants of health and the risk factors shared between oral diseases and other non-communicable diseases.

(Peres et al., 2019)

Closing the gap between deprived and affluent individuals, areas and communities is a difficult task. The first and possibly foremost problem is making available good and free oral health care for all, as several public health measures are implemented earlier or even only in affluent areas and communities that need them least. This recognized theory, proposed in 1971 by Julian Hart and known as the Inverse Care Law, in the beginning was an attack to the private, profit-oriented, healthcare sector which was directed to affluent individuals rather than those in most need, and was thus considered responsible for increasing health inequalities (Hart, 1971). This socialistic view remains true, but was later extended to public health interventions (Watt, 2002), and was also referred to in the inverse equity hypothesis (Victora, Vaughan, Barros, Silva, \& Tomasi, 2000). For example, there is a substantial mismatch in the United States between the territorial distribution of individuals at high lung cancer risk and of centers that perform screening programs with low-dose computerized tomography scan, an expensive measure mostly performed in private clinics (Eberth et al., 2018). The validity of this theory was corroborated by the principal authors of the oral health 2019 Lancet Series. Indeed, Marco A. Peres and his colleagues reported that water fluoridation in Southern Brazil was implemented later in more deprived municipalities with lower child development index and higher illiteracy rate, where caries was more widespread than in more affluent municipalities that were in lower need of water fluoridation (Peres, Fernandes, \& Peres, 2004). Similarly, Richard G. Watt and colleagues analyzed the characteristics of individuals in UK who visit dentists regularly, thus being screened for oral cancer. They specified five oral cancer risk factors and markers and found that the probability of visiting dentists routinely was inversely proportional to the number of these factors and markers (Netuveli, Sheiham, \& Watt, 2006).

The second and more puzzling problem is that individuals and communities at higher disease risk utilize these services least, even within universal health care systems. A comprehensive literature analysis concluded that those who are less likely to attend health checks and screening programs are males, disadvantaged individuals, those with low education and from ethnic minorities, singles, and high risk subjects, such as smokers, alcohol drinkers and obese, who are at a higher risk of developing non-communicable diseases (Dryden, Williams, McCowan, \& Themessl-Huber, 2012).

The literature regarding oral health is full of examples that corroborate this inconceivable aspect of the inverse equity hypothesis (Petti, 2010), as in the episode described by Hannu Hausen during the celebrations of the 50th ORCA Congress in 2003. Hausen was explaining the reasons for caries decline in Finland (Hausen, 2004). Caries prevalence in Finnish children in the 1970s was among the highest in the world. Thus, nationwide free preventive measures were implemented in dental hospitals at no cost for patients, 
including fluoride and fissure sealant. The caries decline was so drastic that in the 1990s, the oral health of Finnish children was among the best in the highly developed countries. For this reason, these programs were progressively discontinued, despite the protests of some dental public health officers. Nevertheless, caries incidence continued to decrease. One of the congress attendees, particularly disappointed, wondered how this could be possible and Hausen's answer was that "fissure sealants were going to the wrong direction", in other words, those who continued to benefit from these free caries preventive programs were those in the least need and programs directed to disadvantaged children were more effective in reducing the overall incidence.

Among other consequences, the inverse equity hypothesis associates with comorbidities and fatalism. Indeed, underprivileged individuals and families frequently suffer many conditions simultaneously, which compromise health and have significant social consequences; the most common of them is psychological distress. "Fatalism is the view that we are powerless to do anything other than what we already do, fatalistic individuals believe that there is nothing they can do to cure or prevent any condition that they are fated to develop, regardless of their level of health literacy" (Petti \& Polimeni, 2011).

\section{3 | FINAL CONSIDERATIONS}

We propose that oral medicine joins the collective effort for renewing oral health and plays a part in revising dentistry and dental teaching. Professionals in the area should bring their expertise to the discussion about upstream policies for the promotion of oral health. Although much has been done to control tobacco and alcohol consumption, much has yet to be done. Hot topics might include the standardization of measurements and methods to assess oral mucosal lesions at the population level; the planning of surveillance systems for premalignant oral lesions and other disorders of interest to oral medicine. The opportunity of screening programs is also a matter deserving of further discussion. Last, but not least, the professional field should consider adhering to the current efforts of integrating oral health into universal health coverage (Fisher, Selikowitz, Mathur, \& Varenne, 2018).

Gender, racial, and socioeconomic inequalities in the incidence of oral diseases and access to dental services remain a global challenge. In 1986, the Ottawa Charter for Health Promotion had considered the social determinants of health. According to this Charter, social justice and equity, along with education and income, a stable ecosystem, sustainable resources, and peace, are among the prerequisites for achieving the goal of health for all. The core of rethinking dentistry and dental teaching for the generations to come lies within the Charter premises. Oral health is not an exclusive responsibility of oral healthcare providers; it is also, and maybe principally, a political, economic, and cultural issue that should move us all.

\section{CONFLICT OF INTEREST}

All authors have none to declare.

\section{AUTHOR CONTRIBUTIONS}

J. L. F. Antunes, A. R. da Cunha, and S. Petti contributed equally in conceiving and conceptualizing the text. All authors approved the final version.

\section{ORCID}

Jose Leopoldo Ferreira Antunes (iD https://orcid. org/0000-0003-3972-9723

Amanda Ramos da Cunha iD https://orcid.

org/0000-0002-6358-5582

Stefano Petti iD https://orcid.org/0000-0001-9996-8860

\section{REFERENCES}

Alnaggar, D., \& Andersson, L. (2015). Emergency management of traumatic dental injuries in 42 countries. Dental Traumatology, 31(2), 8996. https://doi.org/10.1111/edt.12155

Andersson, L., Petti, S., Day, P., Kenny, K., Glendor, U., \& Andreasen, J. O. (2019). Classification, epidemiology and etiology. In J. O. Andreasen, F. M. Andreasen, \& L. Andersson (Eds.), Textbook and color atlas of traumatic injuries to the teeth (5th ed., pp. 251-294). Oxford, UK: Wiley-Blackwell.

Beaglehole, R. H., \& Beaglehole, R. (2019). Promoting radical action for global oral health: Integration or independence? Lancet, 394(10194), 196-198. https://doi.org/10.1016/S0140-6736(19)31610-1

Borum, M. K., \& Andreasen, J. O. (2001). Therapeutic and economic implications of traumatic dental injuries in Denmark: An estimate based on 7549 patients treated at a major trauma centre. International Journal of Paediatric Dentistry, 11(4), 249-258. https:// doi.org/10.1046/j.1365-263X.2001.00277.x

Corrêa-Faria, P., \& Petti, S. (2015). Are overweight/obese children at risk of traumatic dental injuries? A meta-analysis of observational studies. Dental Traumatology, 31(4), 274-282. https://doi.org/10.1111/ edt.12172

Dryden, R., Williams, B., McCowan, C., \& Themessl-Huber, M. (2012). What do we know about who does and does not attend general health checks? Findings from a narrative scoping review. BMC Public Health, 12, 723. https://doi.org/10.1186/1471-2458-12-723

Eberth, J. M., Bozorgi, P., Lebrón, L. M., Bills, S. E., Hazlett, L. J., Carlos, R. C., ... King, J. C. (2018). Geographic availability of low-dose computed tomography for lung cancer screening in the United States, 2017. Preventing Chronic Disease, 15, E119. https://doi.org/10.5888/ pcd15.180241

Erickson, J., Sadeghirad, B., Lytvyn, L., Slavin, J., \& Johnston, B. C. (2017). The scientific basis of guideline recommendations on sugar intake: A systematic review. Annals of Internal Medicine, 166(4), 257-267. https ://doi.org/10.7326/M16-2020

Erickson, J., \& Slavin, J. (2015). Are restrictive guidelines for added sugars science based? Nutrition Journal, 14, 124. https://doi.org/10.1186/ s12937-015-0114-0

Fisher, J., Selikowitz, H. S., Mathur, M., \& Varenne, B. (2018). Strengthening oral health for universal health coverage. Lancet, 392(10151), 899901. https://doi.org/10.1016/S0140-6736(18)31707-0

GBD 2015 Disease and Injury Incidence and Prevalence Collaborators (2016). Global, regional, and national incidence, prevalence, and 
years lived with disability for 310 diseases and injuries, 1990-2015: A systematic analysis for the Global Burden of Disease Study 2015. Lancet, 388(10053), 1545-1602. https://doi.org/10.1016/ S0140-6736(16)31678-6

Glick, M., Williams, D. M., Kleinman, D. V., Vujicic, M., Watt, R. G., \& Weyant, R. J. (2017). A new definition for oral health developed by the FDI World Dental Federation opens the door to a universal definition of oral health. Journal of Public Health Dentistry, 77(1), 3-5. https://doi.org/10.1016/j.ajodo.2016.11.010

Hart, J. T. (1971). The inverse care law. Lancet, 1(7696), 405-412. https:// doi.org/10.1016/s0140-6736(71)92410-x

Hausen, H. (2004). How to improve the effectiveness of caries-preventive programs based on fluoride. Caries Research, 38(3), 263-267. https://doi.org/10.1159/000077765

Kassebaum, N. J., Smith, A., Bernabé, E., Fleming, T. D., Reynolds, A. E., Vos, T., ... Yonemoto, N. (2017). Global, regional, and national prevalence, incidence, and disability-adjusted life years for oral conditions for 195 countries, 1990-2015: A systematic analysis for the global burden of diseases, injuries, and risk factors. Journal of Dental Research, 96(4), 380-387. https://doi.org/10.1177/0022034517 693566

Kearns, C. E., \& Bero, L. A. (2019). Conflicts of interest between the sugary food and beverage industry and dental research organisations: Time for reform. Lancet, 394(10194), 194-196. https://doi. org/10.1016/S0140-6736(19)31277-2

Lingström, P., van Houte, J., \& Kashket, S. (2000). Food starches and dental caries. Critical Reviews in Oral Biology and Medicine, 11(3), 366380. https://doi.org/10.1177/10454411000110030601

Marsh, P. D. (2018). In sickness and in health-what does the oral microbiome mean to us? An ecological perspective. Advances in Dental Research, 29(1), 60-65. https://doi.org/10.1177/0022034517 735295

Moynihan, P., Makino, Y., Petersen, P. E., \& Ogawa, H. (2018). Implications of WHO Guideline on Sugars for dental health professionals. Community Dentistry and Oral Epidemiology, 46(1), 1-7. https://doi. org/10.1111/cdoe.12353

Netuveli, G., Sheiham, A., \& Watt, R. G. (2006). Does the 'inverse screening law' apply to oral cancer screening and regular dental check-ups? Journal of Medical Screening, 13(1), 47-50. https://doi. org/10.1258/096914106776179836

Newbrun, E. (1969). Sucrose, the arch criminal of dental caries. ASDC Journal of Dentistry for Children, 36(4), 239-248.

Peres, M. A., Fernandes, L. S., \& Peres, K. G. (2004). Inequality of water fluoridation in Southern Brazil - the inverse equity hypothesis revisited. Social Science and Medicine, 58(6), 1181-1189. https://doi. org/10.1016/s0277-9536(03)00289-2

Peres, M. A., Macpherson, L. M. D., Weyant, R. J., Daly, B., Venturelli, R., Mathur, M. R., ... Watt, R. G. (2019). Oral diseases: A global public health challenge. Lancet, 394(10194), 249-260. https://doi. org/10.1016/S0140-6736(19)31146-8

Petti, S. (2010). Why guidelines for early childhood caries prevention could be ineffective amongst children at high risk. Journal of Dentistry, 38(12), 946-955. https://doi.org/10.1016/j.jdent.2010.09.002

Petti, S. (2015). Over two hundred million injuries to anterior teeth attributable to large overjet: A meta-analysis. Dental Traumatology, 31(1), 1-8. https://doi.org/10.1111/edt.12126

Petti, S., Andreasen, J. O., Glendor, U., \& Andersson, L. (2018). The fifth most prevalent disease is being neglected by public health organisations. Lancet Global Health, 6, e1070-e1071. https://doi.org/10.1016/ S2214-109X(18)30380-2

Petti, S., Glendor, U., \& Andersson, L. (2018). World traumatic dental injury prevalence and incidence, a meta-analysis-One billion living people have had traumatic dental injuries. Dental Traumatology, 34(2), 71-86. https://doi.org/10.1111/edt.12389

Petti, S., \& Polimeni, A. (2011). Inverse care law. British Dental Journal, 210(8), 343. https://doi.org/10.1038/sj.bdj.2011.291

Pitts, N. B., Zero, D. T., Marsh, P. D., Ekstrand, K., Weintraub, J. A., Ramos-Gomez, F., ... Ismail, A. (2017). Dental caries. Nature Reviews Disease Primers, 3, 17030. https://doi.org/10.1038/nrdp.2017.30

van Houte, J. (1994). Role of micro-organisms in caries etiology. Journal of Dental Research, 73(3), 672-681. https://doi.org/10.1177/00220 345940730031301

van Loveren, C. (2009). Oral and dental health - Prevention of dental caries, erosion, gingivitis and periodontitis. Brussels, Belgium: ILSI Europe.

van Loveren, C., \& Duggal, M. S. (2004). Experts' opinions on the role of diet in caries prevention. Caries Research, 38(Suppl 1), 16-23. https:// doi.org/10.1159/000074358

Victora, C. G., Vaughan, J. P., Barros, F. C., Silva, A. C., \& Tomasi, E. (2000). Explaining trends in inequities: Evidence from Brazilian child health studies. Lancet, 356(9235), 1093-1098. https://doi.org/10.1016/ S0140-6736(00)02741-0

Watt, G. (2002). The inverse care law today. Lancet, 360(9328), 252-254. https://doi.org/10.1016/S0140-6736(02)09466-7

Watt, R. G., Daly, B., Allison, P., Macpherson, L. M. D., Venturelli, R., Listl, S., ... Benzian, H. (2019). Ending the neglect of global oral health: Time for radical action. Lancet, 394(10194), 261-272. https://doi. org/10.1016/S0140-6736(19)31133-X

Zero, D. T. (2004). Sugars - The arch criminal? Caries Research, 38(3), 277-285. https://doi.org/10.1159/000077767

How to cite this article: Antunes JLF, Cunha AR,Petti S. Rethinking dentistry and dental teaching. Oral Dis. 2020;26:6-11. https://doi.org/10.1111/odi.13215 